\title{
First-principles theory of inelastic currents in a scanning tunneling microscope
}

\author{
Stokbro, Kurt; Hu, Ben Yu-Kuang; Thirstrup, C.; Xie, X.C.
}

Published in:

Physical Review B Condensed Matter

Link to article, DOI:

10.1103/PhysRevB.58.8038

Publication date:

1998

Document Version

Publisher's PDF, also known as Version of record

Link back to DTU Orbit

Citation (APA):

Stokbro, K., Hu, B. Y-K., Thirstrup, C., \& Xie, X. C. (1998). First-principles theory of inelastic currents in a scanning tunneling microscope. Physical Review B Condensed Matter, 58(12), 8038-8041.

https://doi.org/10.1103/PhysRevB.58.8038

\section{General rights}

Copyright and moral rights for the publications made accessible in the public portal are retained by the authors and/or other copyright owners and it is a condition of accessing publications that users recognise and abide by the legal requirements associated with these rights.

- Users may download and print one copy of any publication from the public portal for the purpose of private study or research.

- You may not further distribute the material or use it for any profit-making activity or commercial gain

- You may freely distribute the URL identifying the publication in the public portal 


\title{
First-principles theory of inelastic currents in a scanning tunneling microscope
}

\author{
K. Stokbro and Ben Yu-Kuang $\mathrm{Hu}$ \\ Mikroelektronik Centret, Danmarks Tekniske Universitet, Bygning 345ф, DK-2800 Lyngby, Denmark \\ C. Thirstrup * \\ Surface and Interface Laboratory, RIKEN, Saitama 351, Japan \\ X. C. Xie \\ Department of Physics, Oklahoma State University, Stillwater, Oklahoma 74078
}

(Received 27 February 1998)

\begin{abstract}
A first-principles theory of inelastic tunneling between a model probe tip and an atom adsorbed on a surface is presented, extending the elastic tunneling theory of Tersoff and Hamann. The inelastic current is proportional to the change in the local density of states at the center of the tip due to the addition of the adsorbate. We use the theory to investigate the vibrational heating of an adsorbate below a scanning tunneling microscopy tip. We calculate the desorption rate of $\mathrm{H}$ from $\mathrm{Si}(100)-\mathrm{H}(2 \times 1)$ as a function of the sample bias and tunnel current, and find excellent agreement with recent experimental data. [S0163-1829(98)09036-5]
\end{abstract}

Atom manipulation using scanning tunneling microscopes (STM's) has been reported for a wide range of systems. ${ }^{1,2}$ The manipulation of atoms has been attributed to various origins: direct forces between the tip and the surface, ${ }^{3,4}$ indirect interaction through the tip induced electric field, ${ }^{5,6}$ and inelastic scattering by electrons tunneling from the tip to the surface. ${ }^{7,8}$ The last mechanism, for example, is thought to account for the reversible transfer of a Xe atom between a $\mathrm{Ni}(110)$ surface and a $\mathrm{W}$ tip, ${ }^{9-11}$ hydrogen desorption from hydrogen-passivated silicon, ${ }^{7}$ and dissociation of $\mathrm{O}_{2}$ molecules on a $\mathrm{Ni}(110)$ surface $^{8}$ However, it is often not evident from the experimental data which mechanism dominates, and input from theoretical calculations is vital to establish the microscopic mechanisms behind atomic manipulation on surfaces.

In this paper we present a first-principles method for calculating the inelastic current in a STM tunnel junction due to inelastic scattering of the tunneling electrons with an adsorbate-induced resonance. Our method is based on the Tersoff-Hamann approximation for the STM tunnel junction $^{12}$ and builds on previous work on inelastic tunneling in STM's. ${ }^{13-16}$ The main result of this paper, Eq. (9), is an expression for the inelastic current in terms of the partial local density of states (DOS) of the adsorbate wave function at the position of the tip. This expression allows us to calculate the current and bias dependence of the desorption rate of $\mathrm{H}$ from the monohydrate $\mathrm{Si}(100)$ surface, by solving a Pauli master rate equation for transitions between the different vibrational levels of the adsorbate. In a recent letter, ${ }^{17}$ we used Eq. (9) to explain experimental desorption rates at a negative sample bias due to inelastic hole tunneling. Here we present a derivation of Eq. (9), and use it to calculate desorption rates at positive sample bias due to inelastic electron tunneling.

We divide the tunnel region into a tip and a sample part, and assume that the atom to be manipulated is adsorbed onto the sample. The model is shown schematically in Fig. 1. The total Hamiltonian is given by $\hat{H}=\hat{H}_{s+a}+\hat{H}_{\text {tip }}+\hat{H}_{T}$, where the terms on the right are the surface plus adsorbate, tip, and tunneling parts, respectively. We first examine $\hat{H}_{s+a}$. Let $|a\rangle$ and $|k\rangle$ be the one-electron eigenstates of the separated atom and the surface, respectively. When the atom adsorbs onto the surface, a coupling term $\hat{h}_{k a}=\Sigma_{k} t_{k a} \hat{c}_{k}^{\dagger} \hat{a}+$ H.c. is introduced (where $\hat{c}_{k}$ and $\hat{a}$ are the field operators for states $|k\rangle$ and $|a\rangle$, respectively). The surface plus adsorbate system can be diagonalized to yield eigenstates $|\mu\rangle$. The adsorbate also can vibrate on the surface, which implies a localized

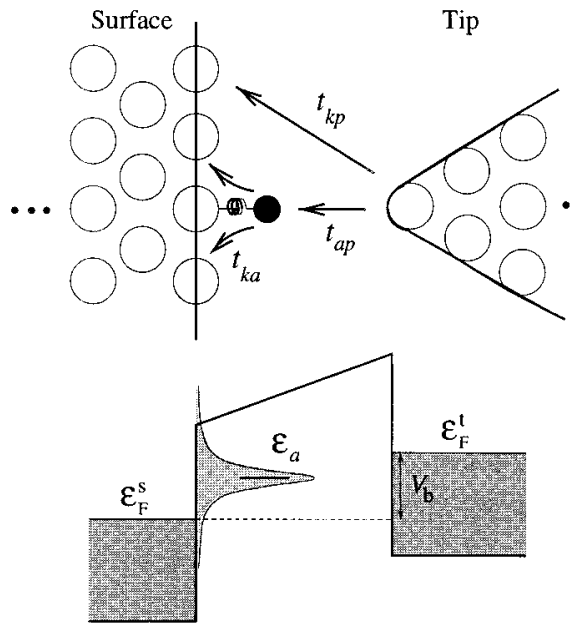

FIG. 1. Schematic figure of the tunneling model (top), and associated density of states (bottom). When the adsorbate (black dot) is separated from the surface, the eigenstates of the surface and adsorbate are $|k\rangle$ and $|a\rangle$, respectively. Upon adsorption, a coupling term with elements $t_{k a}$ is introduced between $|k\rangle$ and $|a\rangle$. In addition, there is a phonon term corresponding to the local oscillation of the adsorbate. The electronic part (i.e., when there are no phonons) can be diagonalized to yield eigenstates $|\mu\rangle$. The center of resonance of the broadened level $|a\rangle, \epsilon_{a}$, is a function of $z$ the distance of the adsorbate from its equilibrium position. 
phonon term with a boson field operator $\hat{b}$. Furthermore, the adsorbate vibration couples to the energy-level of the electron in state $|a\rangle$. Therefore, $\hat{H}_{s+a}=\hat{H}_{e}+\hat{H}_{\mathrm{ph}}+\hat{H}_{e-\mathrm{ph}}$, (the electron, phonon, and coupling term, respectively), where

$$
\begin{gathered}
\hat{H}_{e}=\sum_{\mu} \varepsilon_{\mu} \hat{c}_{\mu}^{\dagger} \hat{c}_{\mu}, \\
\hat{H}_{\mathrm{ph}}=\hbar \omega_{0} \hat{b}^{\dagger} \hat{b}, \\
\hat{H}_{e-\mathrm{ph}}=\lambda\left(\hat{b}^{\dagger}+\hat{b}\right) \hat{a}^{\dagger} \hat{a} .
\end{gathered}
$$

The tip Hamiltonian is given by $\hat{H}_{\text {tip }}=\Sigma_{p} \varepsilon_{p} \hat{c}_{p}^{\dagger} \hat{c}_{p}$ (where $|p\rangle$ are the tip eigenstates) and the tip to surface tunneling by $\hat{H}_{T}=\Sigma_{\mu p} t_{\mu p} \hat{c}_{\mu}^{\dagger} \hat{c}_{p}+$ H.c. $\equiv \Sigma_{\kappa p} t_{\kappa p} \hat{c}_{\kappa}^{\dagger} \hat{c}_{p}+$ H.c. (here $\kappa=k$ or $a$ ). The matrix elements are related via $t_{\kappa p}=\Sigma_{\mu}\langle\kappa \mid \mu\rangle t_{\mu p}$.

The coupling between the tip and the surface is normally weak, hence it is sufficient to work to lowest (i.e., second) order in the tunneling matrix element. Let $|p, n\rangle$ be the state with an electron in state $p$ on the tip and $n$ phonons on the adsorbate, and $\left|\mu, n^{\prime}\right\rangle$ be the coupled eigenstate of $H_{s}$, arising from the interaction between electron state $\mu$ and $n^{\prime}$ adsorbate phonons. The inelastic component of the current from tip to surface which changes the phonon occupation from $n$ to $n^{\prime}$ is given by ${ }^{18}$

$$
\begin{aligned}
I\left(n \rightarrow n^{\prime}\right)= & \frac{2 \pi e}{\hbar} \sum_{\mu p} f_{t}\left(\varepsilon_{p}\right)\left[1-f_{s}\left(\varepsilon_{\mu}\right)\right] \\
& \times\left|T_{p n, \mu n^{\prime}}\right|^{2} \delta\left[\varepsilon_{p}-\varepsilon_{\mu}-\left(n^{\prime}-n\right) \hbar \omega_{0}\right],
\end{aligned}
$$

where $T_{p n, \mu n^{\prime}}=\left\langle p n\left|\hat{H}_{T}\right| \mu n^{\prime}\right\rangle$ is the $T$-matrix element and $f_{s, t}(\varepsilon)=1 /\left\{1+\exp \left[\left(\varepsilon-\varepsilon_{F s, F t}\right)\right]\right\}$ are Fermi distribution functions. The Fermi levels $\varepsilon_{F s}$ and $\varepsilon_{F t}$ of the sample and tip, respectively, are determined by the applied sample bias, $V_{b}$, and related by $\varepsilon_{F s}=\varepsilon_{F t}-e V_{b}$.

We define uncoupled electron-phonon eigenstates (i.e., setting $\lambda=0)$ of the sample as $|\mu\rangle \otimes|n\rangle$. The coupled eigenstates can be expressed as a Born series ${ }^{19,20}$

$$
|\mu, n\rangle=\sum_{l=0}^{\infty}\left[\hat{G}_{\otimes}\left(\epsilon_{\mu, n}\right) \hat{H}_{e-\mathrm{ph}}\right]^{l}|\mu\rangle \otimes|n\rangle,
$$

where $\epsilon_{\mu, n} \approx \varepsilon_{\mu}+n \hbar \omega_{0}$ is the eigenenergy of $|\mu, n\rangle$, and $\hat{G}_{\otimes}=\left(\varepsilon-\hat{H}_{e}-\hat{H}_{\mathrm{ph}}+i 0^{+}\right)^{-1}$ is the Green function of the uncoupled electron-phonon system. Using Eq. (3), the $T$-matrix elements can be written as

$$
\begin{aligned}
T_{p n, \mu n^{\prime}}= & \sum_{\mu^{\prime}} t_{\mu^{\prime} p}^{*} \sum_{l=0}^{\infty}\left\langle\mu^{\prime}\right| \otimes\left\langle n\left|\left[\hat{G}_{\otimes}\left(\epsilon_{\mu, n^{\prime}}\right) \hat{H}_{e-\mathrm{ph}}\right]^{l}\right| \mu\right\rangle \\
& \otimes\left|n^{\prime}\right\rangle .
\end{aligned}
$$

We now consider $n^{\prime}=n+N$; i.e., where $N$ phonons are absorbed (the emission case is similar). The first term in the Born series [Eq. (4)] which contributes is the $N$ th-order term containing $\left(\hat{b}^{\dagger}\right)^{N}$. In this paper, we ignore the higher-order terms in the series. ${ }^{21}$ This term gives

$$
\begin{aligned}
T_{p n, \mu n+N}^{(N)}= & \sqrt{(N+n) ! / n !} \lambda^{N}\langle a \mid \mu\rangle \sum_{\mu^{\prime}} t_{\mu^{\prime} p}^{*} \\
& \times\left\langle\mu^{\prime}\left|\hat{g}\left(\varepsilon_{\mu}+N \hbar \omega_{0}\right)\right| a\right\rangle \\
& \times \prod_{j=1, N-1}\left\langle a\left|\hat{g}\left(\epsilon_{\mu, m}+(N-j) \hbar \omega_{0}\right)\right| a\right\rangle
\end{aligned}
$$

where $\hat{g}(\varepsilon)=\left(\varepsilon-\hat{H}_{e}+i 0^{+}\right)^{-1}$ is the sample plus adsorbate electron Green function (without phonons).

The Lippmann-Schwinger equation gives the one-electron eigenstates of the sample plus adsorbate in terms of the eigenstates of the atom and the sample, $\left|\mu_{k}\right\rangle=|k\rangle$ $+\hat{g}\left(\varepsilon_{\mu_{k}}\right)|a\rangle t_{k a}^{*}$. This, together with Eq. (5) and the relationship between $t_{\mu p}$ and $t_{\kappa p}$, yields

$$
\begin{aligned}
T_{p n, \mu_{k} n+N}^{(N)}= & \sqrt{(n+N) ! / n !} \lambda^{N}\left[t_{\mu_{k} p}^{*}-t_{k p}^{*}\right] \\
& \times \prod_{j=0, N-1}\left\langle a\left|\hat{g}\left(\varepsilon_{\mu_{k}}+(N-j) \hbar \omega_{0}\right)\right| a\right\rangle \\
& +\Upsilon_{p n, \mu_{k} n+N}^{(N)} .
\end{aligned}
$$

The term $Y$ corresponds to processes where the electron tunnels from the tip into the sample state, $k$, hops back into the adsorbate, emits phonons, and hops back out again. Generally, such terms are small, and moreover it may even be inaccurate to include this term, since the lifetime of state $k$ probably will be dominated by scattering with electron-hole excitations or bulk-phonon states (which is excluded in our model).

The next step is to relate the above equation to the projectors $\langle a \mid \mu\rangle$. We use the identity

$$
|\langle a|\hat{g}(\varepsilon)| a\rangle|^{2} \equiv \frac{\pi \sum_{\mu^{\prime}}\left|\left\langle\mu^{\prime} \mid a\right\rangle\right|^{2} \delta\left(\varepsilon_{\mu^{\prime}}-\varepsilon\right)}{\Delta(\varepsilon)},
$$

where $\Delta(\varepsilon)$ is the magnitude of the imaginary part of the self-energy of $\langle a|\hat{g}(\varepsilon)| a\rangle$. We now assume that the adsorbate resonance is broad compared to the $\hbar \omega_{0}$. Hence we (1) approximate the terms $\varepsilon_{\mu_{k}}+(N-j) \hbar \omega_{0} \approx \varepsilon_{\mu_{k}}$, (2) assume $\Delta(\varepsilon) \approx \Delta\left(\widetilde{\varepsilon}_{a}\right) \equiv \Delta$, and (3) set $\Sigma_{\mu^{\prime}}\left|\left\langle\mu^{\prime} \mid a\right\rangle\right|^{2} \delta\left(\varepsilon_{\mu^{\prime}}-\varepsilon_{\mu}\right)$ $\approx|\langle\mu \mid a\rangle|^{2} \bar{\rho}_{s}$ where $\bar{\rho}_{s}=\Sigma_{\mu^{\prime}} \delta\left(\varepsilon_{\mu}-\widetilde{\varepsilon}_{a}\right)$ is the density of states at $\tilde{\varepsilon}_{a}$. These assumptions together, with Eqs. (2), (6) and (7), yield

$$
\begin{aligned}
I(n \rightarrow n+N)= & \frac{(n+N) !}{n !} \frac{2 \pi e}{\hbar} K^{N} \sum_{p \mu_{k}}\left|t_{\mu_{k} p}-t_{k p}\right|^{2} \\
& \times\left|\left\langle\mu_{k} \mid a\right\rangle\right|^{2 N} f_{t}\left(\varepsilon_{p}\right)\left[1-f_{s}\left(\varepsilon_{\mu_{k}}\right)\right] \\
& \times \delta\left(\varepsilon_{p}-\varepsilon_{\mu_{k}}-N \hbar \omega\right),
\end{aligned}
$$

where $K=\pi \bar{\rho}_{s} \lambda^{2} / \Delta$ is a dimensionless quantity.

The last step is to evaluate the matrix element $t_{\mu_{k} p}-t_{k p}$ using the Tersoff-Hamann approximation. ${ }^{12,22}$ In this approximation the tunneling is assumed to be through a single atom at the tip apex with an $s$-type wave function. We define $\left|t_{\mu_{k} p}-t_{k p}\right|^{2} \equiv f_{\mu_{k} p}\left|t_{\mu_{k} p}\right|^{2}$, where $f_{\mu_{k}} \equiv\left|\left\langle a \mid \mu_{k}\right\rangle\right|^{2} /\left(x_{\mu_{k}}\right.$ 
$\left.+\left|\left\langle a \mid \mu_{k}\right\rangle\right|^{2}\right)$ is essentially the fraction of electrons which tunnel from the tip into state $\mu_{k}$ through the resonance. The evaluation of $\left|t_{\mu_{k} p}-t_{k p}\right|^{2}$ is then equivalent to previous work on elastic tunneling, ${ }^{22}$ and for a $\mathrm{W}$ tip we obtain

$$
\begin{aligned}
I(n \rightarrow n+N) \approx & C_{\mathrm{W}} \frac{(n+N) !}{n !} \int_{N \hbar \omega_{0}}^{e V_{b}}\left|e^{2 R_{\mathrm{W}} \kappa(\varepsilon)}\right| \\
& \times \rho_{N}\left(d+R_{\mathrm{W}}, \varepsilon, E\right) d \varepsilon .
\end{aligned}
$$

In this equation $d$ is the tip-sample distance, $\kappa(\varepsilon)$ $=\sqrt{2 m\left(\phi_{t}+e V_{b}-\varepsilon\right)} / \hbar$ is the wave-function inverse decay length, $\phi_{t}=4.5 \mathrm{eV}$ the work function of the $\mathrm{W}$ tip ${ }^{24} E$ is the electric field between the tip and sample, $R_{\mathrm{W}}=3 \mathrm{bohr}$ is the atomic radius of $\mathrm{W}$, and the normalization constant $C_{\mathrm{W}}$ $=0.007 R_{\mathrm{W}}^{2}$ ampere bohr is obtained from a calculation of a model $\mathrm{W}$ tip. ${ }^{23}$ The local weighted DOS,

$$
\rho_{n}(\mathbf{r}, \varepsilon, E)=K^{n} \sum_{\mu} f_{\mu}|\langle a \mid \mu\rangle|^{2 n}\left|\psi_{\mu}(\mathbf{r}, E)\right|^{2} \delta\left(\varepsilon-\varepsilon_{\mu}\right),
$$

is the DOS weighted by $n$ powers of the dimensionless coupling $K$ and the projection $\langle a \mid \mu\rangle$ of the resonance wave function $\psi_{a}$ onto the eigenstates $\psi_{\mu}$ of the sample. For high biases $\left(\left|V_{b}\right|>2 \mathrm{~V}\right)$ the effect of the electric field between the tip and the sample, $E$, must be taken into account when calculating $\psi_{\mu} \cdot{ }^{23}$ The inelastic current with energy transfer $n \hbar \omega_{0}$ is weighted by $\left(|\langle a \mid \mu\rangle|^{2} K\right)^{n}$; thus only eigenstates with a significant overlap with the adsorbate resonance contribute to the inelastic current.

We now use Eq. (9) to model the STM-induced desorption of $\mathrm{H}$ from $\mathrm{Si}(100)-\mathrm{H}(2 \times 1)$. It has previously been proposed $^{7}$ that inelastic scattering of the tunneling electrons with $\mathrm{Si}-\mathrm{H} 6 \sigma^{*}$ resonance is the dominant desorption mechanism for sample biases in the range $2 \mathrm{~V}<V_{b}<4 \mathrm{~V}$. In the following we make a first-principles calculation of the desorption rate due to this mechanism as a function of the tunnel current and sample bias, and compare with experimental desorption rates.

The electronic structure calculations are based on densityfunctional theory ${ }^{25,26}$ within the generalized-gradient approximation ${ }^{27}$ using 20 -Ry plane-wave basis sets. We describe the $\mathrm{Si}(100)-\mathrm{H}(2 \times 1)$ surface by a 12 -layer $2 \times 1$ slab, and use $32 k$ points in the surface Brillouin zone. Ultrasoft pseudopotentials ${ }^{28}$ are used for both $\mathrm{H}$ and Si. The geometry of the surface is obtained by relaxing the $\mathrm{H}$ atoms and the first six Si layers, and the resulting bond lengths and bond angles of the surface atoms are similar to other first principles calculations. ${ }^{29,30}$ From a frozen phonon calculation we obtain the Si-H stretch frequency $\hbar \omega_{0}=0.26 \mathrm{eV}$, and subtracting the ground-state energy of a free $\mathrm{H}$ atom we calculate the desorption barrier $E_{\mathrm{des}}=3.36 \mathrm{eV}$.

We will use the Si-H molecular $6 \sigma^{*}$ eigenstate, $\left|m_{6 \sigma^{*}}\right\rangle$, to represent the $6 \sigma^{*}$ resonance. We expect $\left|m_{6 \sigma^{*}}\right\rangle$ to have a large overlap with the $6 \sigma^{*}$ resonance, and smaller overlaps with the other resonances localized around $\mathrm{Si}-\mathrm{H}$, which are mainly the $\mathrm{Si}-\mathrm{H} 4 \sigma$ and $5 \sigma$ resonances. The $4 \sigma$ and $5 \sigma$ resonances are well below the Fermi level, and we remove these components by projecting out the overlap between $\left|m_{6 \sigma^{*}}\right\rangle$ and occupied slab eigenstates. In Fig. 2 we show the resulting $6 \sigma^{*}$ partial DOS for different Si-H bond lengths $\Delta z$.

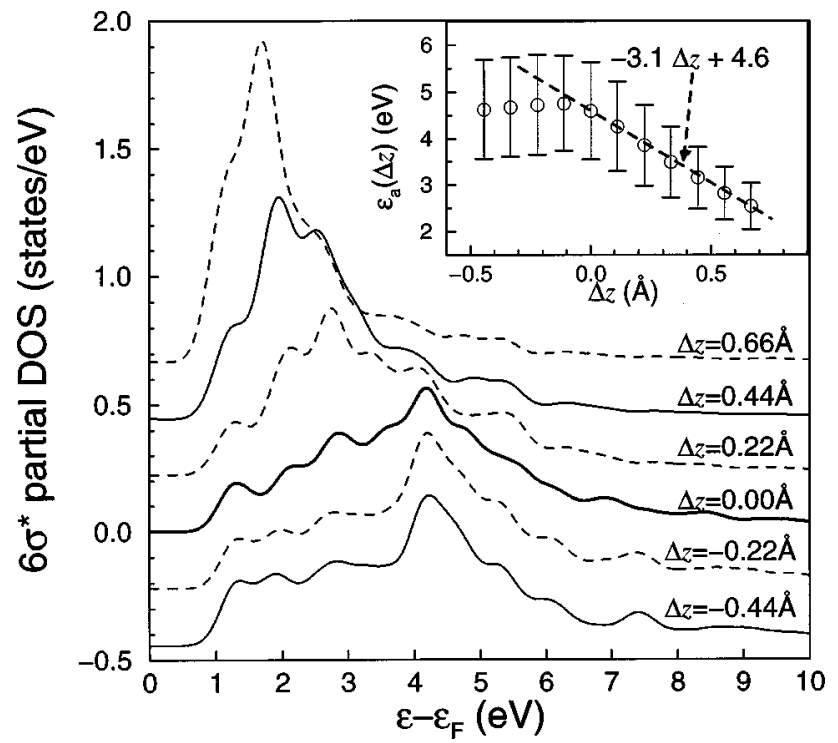

FIG. 2. Projections of the Si-H molecular $6 \sigma^{*}$ eigenstate onto the eigenstates, $\mu$, of a $\mathrm{Si}(100)-\mathrm{H}(2 \times 1)$ slab for different $\mathrm{Si}-\mathrm{H}$ bond lengths ( $\Delta z=0$ is the equilibrium bond lengths). Only projections of states with $\epsilon_{\mu}>\epsilon_{F}$ are included, and each partial DOS is normalized to 1 . The inset shows the average centers (circle) and widths (error bar) as determined from first and second moments. The dashed line shows the results of a linear least-squares fit to centers, $\epsilon_{a}$, with $z>-0.1 \AA$.

From the first and second moments we determine the average centers, $\widetilde{\varepsilon}_{a}(\Delta z)$, and widths, $\Delta(\Delta z)$, as shown in the inset of Fig. 2. At the equilibrium bond length, $\Delta z=0$, the resonance is centered at $\widetilde{\varepsilon}_{a}=4.6 \mathrm{eV}$, and has an average width $\Delta$ $=1 \mathrm{eV}$. For $\Delta z>-0.1 \AA$, the variation of $\widetilde{\varepsilon}_{a}$ with $\Delta z$ is nearly linear and from a linear, least squares fit in this region we calculate $\partial \varepsilon_{a} / \partial z=-3.12 \pm 0.06 \mathrm{eV} / \AA$. The electronphonon coupling term is given by $\lambda=\sqrt{\hbar /\left(2 M \omega_{0}\right)} \partial \varepsilon_{a} / \partial z$, where $M$ is the adsorbate mass, and from the above value of $\partial \varepsilon_{a} / \partial z$ we obtain $\lambda=0.26 \mathrm{eV}$.

To find $\bar{\rho}_{s}$ we use the relation $n_{a}(\varepsilon) \approx|\langle a \mid \mu\rangle|^{2} \bar{\rho}_{s}$. Considering only the eigenstates with the largest projections $\langle a \mid \mu\rangle$, we find $\bar{\rho}_{s} \approx 1.5 \mathrm{eV}^{-1}$. To estimate an average value of $x_{\mu}$, we will use the relation $\left|t_{\mu p}\right|^{2} \approx\left(|\langle\mu \mid a\rangle|^{2}\right.$ $\left.+x_{\mu}\right)\left|t_{a p}\right|^{2}$. We first select the state, $\beta$, with largest overlap, $\langle a \mid \beta\rangle$, in the energy range of interest. If we assume $\left|t_{a p}\right|^{2}$ and $x_{\mu}$ are constant in the energy range, we obtain the equation $x_{\mu}=\left(|\langle\beta \mid a\rangle|^{2}\left|t_{\mu p}\right|^{2}-|\langle\mu \mid a\rangle|^{2}\left|t_{\beta p}\right|^{2} /\left|t_{\beta p}\right|^{2}-\left|t_{\mu p}\right|^{2}\right)$, which we use to estimate $x$ for each eigenstate in the energy range $2-4 \mathrm{~V}$. We find the average value $x \approx 0.01$ $\left(\sim 0.1|\langle a \mid \beta\rangle|^{2}\right)$. With this value we obtain that $I_{0} / I \approx 0.5$ for biases in the range $2-4 \mathrm{~V}$; thus $50 \%$ of the elastic electrons passes through the resonance.

We now have all the parameters entering Eq. (9) for the inelastic current. From the inelastic currents we calculate the desorption rate $R$ by solving the Pauli master equation for the transitions among the various levels of the oscillator, ${ }^{10}$ and vibrational relaxations due to phonon-phonon coupling is described by a current independent relaxation rate, $\gamma=1$ $\times 10^{8} \mathrm{~s}^{-1}$. ${ }^{7,31} \mathrm{We}$ assume that desorption occurs when the energy of the $\mathrm{H}$ atom exceeds the desorption energy $E_{\text {des }}$ $=3.36 \mathrm{eV}$, corresponding to a truncated harmonic potential well with 13 levels. Our objective is to obtain $R$ as function of $I$ and $V_{b}$. However, the inelastic current is a function of 
$V_{b}$ and $d$, and we therefore calculate the elastic current $I\left(V_{b}, d\right)$ to establish a relation between $I, V_{b}$, and $d$. For a given value of $d$ and $V_{b}$ we then calculate the inelastic currents $I(n \rightarrow n+N)$ and $I(n+N \rightarrow n)$ for all bound vibrational states $n=1,2, \ldots, 13$. We include events with $N=1,2$, and 3, and note that the $N=1$ term gives the main contribution to the desorption rate in the current range relevant for desorption.

In Fig. 3 we compare the calculated current dependent desorption rate for sample biases of $2,2.5$, and $3 \mathrm{~V}$ with experimental data obtained by us (open symbols) and Shen et $a .^{7}$ (filled symbols). The two experimental data sets have been obtained on highly doped $n$-type samples using similar experimental procedures. The dashed lines show the firstprinciples calculation of the desorption rates. Both experimental and theoretical desorption rates follow a power law $R \propto I^{\alpha}$, with $\alpha \sim 9-10$ for the theoretical curves and $\alpha \sim 10-13$ for the experimental data. However, the theoretical curves are shifted toward lower currents. The discrepancy is due to the three main approximations of the theoretical model: an expansion based on $\Delta \gg \lambda, \omega_{0}$, a harmonic approximation for the $\mathrm{Si}-\mathrm{H}$ bond potential, and neglect of excited-state orbital relaxation when calculating the resonance wave function. In order to obtain theoretical desorption rates comparable to experiment, we adjust the electronphonon coupling. Solid lines in Fig. 3 shows results using $\lambda=0.20 \mathrm{eV}$, and we see that with this moderate change of the coupling constant we can obtain quantitative agreement with the experimental data in the voltage range $2-3 \mathrm{~V}$. Above 3 $\mathrm{V}$, the calculated desorption rates are too small to explain experimental desorption rates, and the measured desorption rates must be related to another mechanism, most likely direct excitation of the Si-H bond as suggested in Ref. 7.

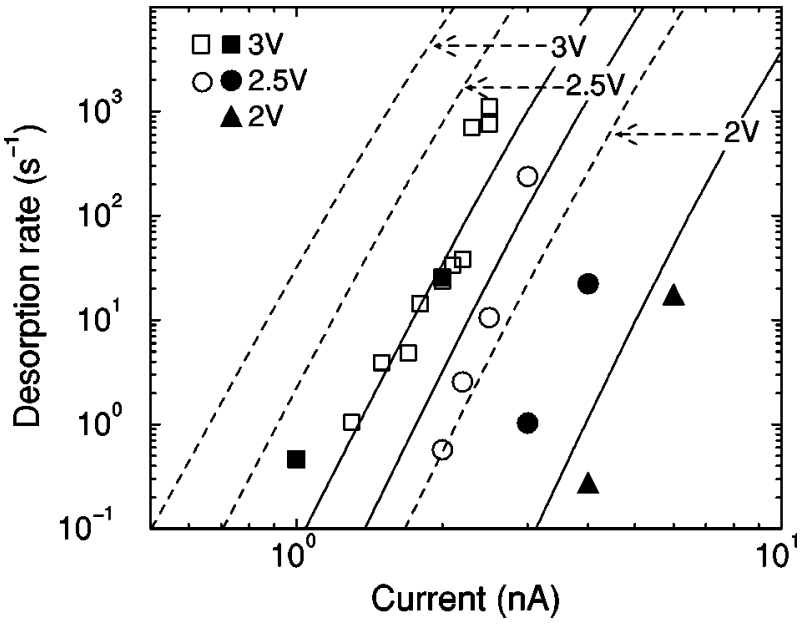

FIG. 3. Desorption rate $R$ as a function of current for $V_{b}$ $=3.0 \mathrm{~V}$ (square), $V_{b}=2.5 \mathrm{~V}$ (circles), and $2.5 \mathrm{~V}$ (triangles). Open symbols show our experimental data, and filled symbols show data from Ref. 7. Dashed lines show theoretical calculations with first principles value $\lambda=0.26 \mathrm{eV}$ for the electron-phonon coupling, while solid lines show results using a fitted value $\lambda=0.20 \mathrm{eV}$.

In conclusion, we have presented a first-principles theory of inelastic scattering, and used it to calculate the voltage and current dependent variation of the hydrogen desorption rate from the $\mathrm{Si}(100)-\mathrm{H}(2 \times 1)$ surface at positive bias conditions. We find that the desorption in the voltage range $2-3 \mathrm{~V}$ is consistent with vibrational heating of the $\mathrm{H}$ atom due to inelastic scattering with the $\mathrm{Si}-\mathrm{H} 6 \sigma^{*}$ resonance.

This work was supported by the Japanese Science and Technology Agency, and the use of Danish national computer resources was supported by the Danish Research Councils. B.Y.-K.H. and X.C.X. acknowledge a grant from NATO.
*Present address: Vir-tech, Kuldyssen 10, DK-2630 Tåstrup, Denmark.

${ }^{1}$ J. A. Stroscio and D. M. Eigler, Science 254, 1319 (1991).

${ }^{2}$ P. Avouris, Acc. Chem. Res. 28, 95 (1995).

${ }^{3}$ D. M. Eigler and E. Schweizer, Nature (London) 344, 524 (1990).

${ }^{4}$ L. Bartels, G. Meyer, and K.-H. Rieder, Phys. Rev. Lett. 79, 697 (1997)

${ }^{5}$ L. J. Whitman, J. A. Stroscio, R. A. Dragoset, and R. J. Celotta, Science 251, 1206 (1991).

${ }^{6}$ H. Uchida, D. Huang, F. Grey, and M. Aono, Phys. Rev. Lett. 70, 2040 (1993).

${ }^{7}$ T.-C. Shen et al., Science 268, 1590 (1995).

${ }^{8}$ B. C. Stipe et al., Phys. Rev. Lett. 78, 4410 (1997).

${ }^{9}$ D. M. Eigler, C. P. Lutz, and W. E. Rudge, Nature (London) 352, 600 (1991).

${ }^{10}$ S. Gao, M. Persson, and B. I. Lundqvist, Solid State Commun. 84, 271 (1992).

${ }^{11}$ M. Brandbyge and P. Hedegård, Phys. Rev. Lett. 72, 2302 (1994).

${ }^{12}$ J. Tersoff and D. R. Hamann, Phys. Rev. B 31, 805 (1985).

${ }^{13}$ S. Gao, Phys. Rev. B 55, 1876 (1997).

${ }^{14}$ S. Gao, M. Persson, and B. I. Lundqvist, Phys. Rev. B 55, 4825 (1997).

${ }^{15}$ G. P. Salam, M. Persson, and R. E. Palmer, Phys. Rev. B 49, 10655 (1994).
${ }^{16}$ B. N. J. Persson, Phys. Ser. 38, 282 (1988).

${ }^{17}$ K. Stokbro et al., Phys. Rev. Lett. 80, 2618 (1998).

${ }^{18}$ G. D. Mahan, Many Particle Physics (Plenum, New York, 1990).

${ }^{19}$ B. A. Lippmann and J. Schwinger, Phys. Rev. 79, 469 (1950).

${ }^{20}$ L. I. Schiff, Quantum Mechanics (McGraw-Hill, Singapore, 1968).

${ }^{21}$ The higher-order terms are of order $\left|T^{N+m} / T^{N}\right|^{2}=O\left[(\lambda / \Delta)^{2 m}\right]$, and can usually be neglected. If needed, these terms can also be relatively easily calculated.

${ }^{22}$ C. J. Chen, Introduction to Scanning Tunneling Microscopy (Oxford University Press, New York, 1993).

${ }^{23}$ K. Stokbro, U. Quaade, and F. Grey, Appl. Phys. A: Mater. Sci. Process. 66, 5907 (1998).

${ }^{24}$ CRC Handbook of Chemistry and Physics, 75th ed., edited by D. R. Lide (CRC Press, New York, 1994).

${ }^{25}$ P. Hohenberg and W. Kohn, Phys. Rev. 136, B864 (1964).

${ }^{26}$ W. Kohn and L. J. Sham, Phys. Rev. 140, A1133 (1965).

${ }^{27}$ J. P. Perdew et al., Phys. Rev. B 46, 6671 (1992).

${ }^{28}$ D. Vanderbilt, Phys. Rev. B 41, 7892 (1990).

${ }^{29}$ P. Kratzer, B. Hammer, and J. K. Nørskov, Phys. Rev. B 51, 13432 (1995).

${ }^{30}$ K. R. Radeke and E. A. Carter, Phys. Rev. B 54, 11803 (1996).

${ }^{31}$ P. Guyot-Sionnest, P. H. Lin, and E. M. Hiller, J. Chem. Phys. 102, 4269 (1995). 